\title{
Estrategias de comercialización para mejorar la cadena de valor de la artesanía en la Región Amazonas, 2019
}

\section{Marketing strategies to improve the value chain of craftsmanship in the Amazonas- Region, 2019}

\author{
Robert Frank Rituay Yoplac ${ }^{1}$; Mscm. Yuri Reyna Marín² ;Ing. John Alexander Torrejon Llaja ${ }^{3}$
}

\section{RESUMEN}

El objetivo de esta investigación fue plantear estrategias de comercialización para mejorar la cadena de valor de la artesanía en la región Amazonas, buscando así mejorar el nivel de competitividad de los artesanos y de todos los agentes involucrados; debido a la problemática principal que ha sido los inadecuados canales de comercialización. El tipo de investigación desarrollada fue con diseño descriptivo de tipo transversal, debido a que la recolección de datos se realizó en un solo momento de tiempo teniendo como población para la investigación a artesanos, comercializadores y clientes; como resultados producto del diagnóstico la artesanía en la Región Amazonas tiene bajo nivel de diseño e innovación, falta de identidad cultural y uso de iconografía local, bajo nivel de mejora de procesos de producción e inadecuados canales de comercialización; a consecuencia de esto se desarrollaron estrategias de mejora, las principales fueron fortalecimiento de capacidades en diseño e innovación del producto, uso y aplicación de nuevos procesos y técnicas para la elaboración de sus productos, con el apoyo de las instituciones involucradas(CITE Utcubamba) a través de un manual de procesos y un manual de uso y aplicación de iconografías para artesanía textil.

Palabras clave: Estrategias, comercialización, cadena de valor, marketing, artesanía.

\begin{abstract}
The objective of this research was to propose marketing strategies to improve the value chain of handicrafts in the Amazon region, thus seeking to improve the level of competitiveness of artisans and all the agents involved; due to the main problem that has been the inadequate marketing channels. The type of research carried out was with a descriptive design of a transversal type, because the data collection was carried out in a single moment of time having artisans, marketers and customers as research population; As a result of the diagnosis, handicrafts in the Amazon Region have a low level of design and innovation, lack of cultural identity and use of local iconography, low level of improvement of production processes and inadequate commercialization channels; as a result, improvement strategies were developed, the main ones were capacity building in product design and innovation, use and application of new processes and techniques for the elaboration of their products, with the support of the institutions involved (CITE Utcubamba) to through a manual of processes and a manual of use and application of iconographies for textile crafts.
\end{abstract}

Keywords: Strategies, marketing, value chain, marketing, crafts.

\footnotetext{
Bachiller en Ingeniería en Agronegocios, Universidad Nacional Toribio Rodríguez de Mendoza de Amazonas. Correo electrónico:frankry97@gmail.com

${ }^{2}$ Ingeniero Industrial, Profesor Principal Tiempo Completo de la Facultad de ingeniería zootecnista, agronegocios y biotecnología de la Universidad Nacional Toribio Rodríguez de Mendoza de Amazonas. Correo electrónico: yuri.reina@gmail.com

${ }^{3}$ Ingeniero Agroindustrial, Coordinador de proyectos del Centro de innovación Tecnológica de artesanía y Turismo CITE Utcubamba. Correo electrónico: alexander.jatll@gmail.com
} 


\section{INTRODUCCIÓN}

La comercialización es uno de los eslabones importantes de la cadena productiva de la artesanía debido a que la mayoría de productos artesanales tienen un mercado dinámico; las estrategias de comercialización ayudan a alcanzar determinados objetivos relacionados con la mercadotecnia, tales como dar a conocer un nuevo producto, aumentar las ventas o lograr una mayor participación en el mercado, posicionamiento de la marca y mejorar el nivel socioeconómico de los artesanos. La artesanía en la región Amazonas es un sector que se debe visualizar como una oportunidad de negocio, ya que existen artesanos que elaboran productos de excelente calidad, los cuales destacan las líneas de bisutería, textil, madera y cerámica que son ofrecidos a los turistas locales, nacionales e internacionales debido a que el turismo está relacionado directamente con la compra de artesanías; así mismo el Centro de Innovación Tecnológica de Artesanía y Turismo (2018) la compra de artesanía complementa al motivo de viaje, ligado siempre a un interés cultural o de tener un recuerdo del lugar visitado, se trata de un medio que genera beneficios para los artesanos, con la relativamente escasa participación de intermediarios y por lo tanto de gran interés para el país, Ministerio de Comercio Exterior y Turismo (2018) refiere que en los últimos años en la Región Amazonas se registra un importante crecimiento anual del turismo del $18 \%$, estos provinieron principalmente de Alemania, Estados Unidos, Francia y España. A pesar de que hay mercado potencial, no se ha logrado encaminar la artesanía con un enfoque

empresarial, por lo que se hizo necesario e imprescindible el presente trabajo de investigación.

\section{MATERIAL Y MÉTODOS}

El tipo de investigación que se desarrolló fue; descriptiva a lo que refiere Abreu (2014) que en este método se realiza una exposición narrativa, numérica y/o gráfica, bien detallada y exhaustiva de la realidad que se estudia, a través de ello se caracterizó la artesanía de la región Amazonas, conociendo así los principales actores que forman parte de la cadena de valor, identificando los puntos críticos. También se desarrolló una investigación de diseño transversal, a lo que refiere Hernández, F. (2003) cuando la recolección de datos se realiza en un solo momento de tiempo.

Para el desarrollo de la presente investigación el universo de la población está conformado por los diferentes actores de la cadena de valor de artesanía de la Región Amazonas, que va desde productores (asociaciones), comercializadores (tiendas artesanales) y clientes (Turistas).

Por lo tanto, la muestra para el eslabón de producción está conformado por 50 artesanos que pertenecen a las siguientes asociaciones como son Asociación para el Desarrollo Chaqapampa, Asociación de artesanía, "María Auxiliadora", Asociación Tishana Gocta, y Asociación Comunal Productiva Tejedoras de María, dichas asociaciones fueron seleccionadas debido a que están ubicadas en puntos estratégicos que son los principales destinos turísticos de la región.

Así mismo para el eslabón de comercialización está conformado por 50 trabajadores que pertenecen a 22 tiendas que están ubicadas en las

3 provincias como son Luya, Bongará y Chachapoyas, estas tiendas artesanales fueronseleccionadas debido a que están ubicadas en los puntos estratégicos del turismo.

Finalmente, el eslabón de los clientes está conformado por 50 clientes o consumidores que fueron encuestados en los principales atractivos turísticos como Kueláp y Gocta.

Dichas muestras fueron seleccionadas a criterio y a conveniencia del investigador. Para la recopilación de la información se utilizó como técnica la encuesta y como instrumento el cuestionario que fue elaborado por el autor, así mismo validado por expertos y a través de la puntuación del alfa de Cronbach.

\section{RESULTADOS}

\subsection{Análisis Estratégico de la Cadena Productiva} de Artesanía

Tabla 1: Línea artesanal que más se produce.

\begin{tabular}{ccc}
\hline & Frecuencia & Porcentaje \\
\hline Textil & 40 & $80 \%$ \\
Cerámica & 1 & $2 \%$ \\
Bisutería & 3 & $5 \%$ \\
Madera & 6 & $13 \%$ \\
Total & 50 & $100 \%$
\end{tabular}

En la tabla 1 se observa que; la artesanía en la región Amazonas está dividida en 4 principales líneas, como son artesanía textil, madera, bisutería y cerámica, siendo la línea artesanal que más producen las asociaciones encuestadas es la línea de artesanía textil.

Tabla 2: Línea de artesanía que más se comercializa

\begin{tabular}{ccc}
\hline & Frecuencia & Porcentaje \\
\hline Artesanía Textil & 19 & $38.6 \%$ \\
Artesanía en & 13 & $27.1 \%$ \\
Madera & & \\
Artesanía en & 11 & $20.7 \%$ \\
Bisutería & & \\
\hline
\end{tabular}




\begin{tabular}{ccc}
\hline $\begin{array}{c}\text { Artesanía en } \\
\text { Cerámica } \\
\text { Total }\end{array}$ & 7 & $13.6 \%$ \\
\hline
\end{tabular}

En la tabla 2 se observa que la artesanía textil es la que más se vende representada con un $38.6 \%$, con un $27.1 \%$ son productos de madera, el 20.7

$\%$ de ellas son de bisutería y el $13.6 \%$ de los productos son de cerámica.

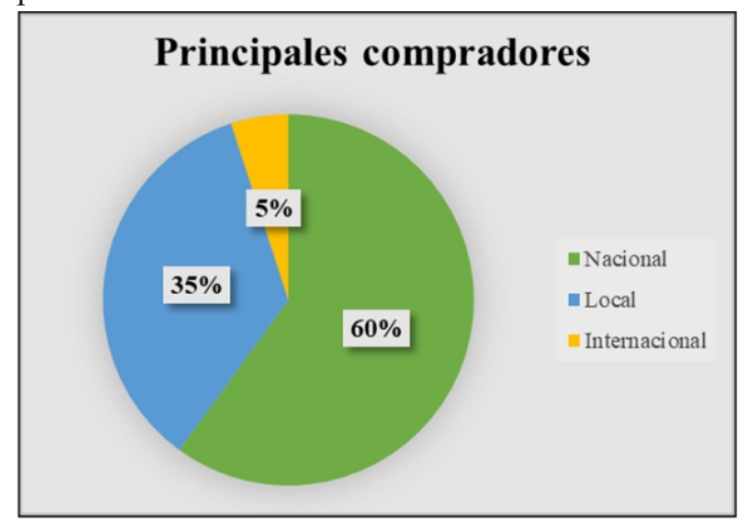

Figura 1: Principales compradores

En la figura 1 se observa que los principales compradores de artesanía con un $60 \%$ son de procedencia nacional, esto se debe a que la gran mayoría son turistas, quienes durante su visita a los atractivos turísticos de la región adquieren artesanías, con un $35 \%$ los clientes son de procedencia local y con un $5 \%$ son de procedencia internacional.

\section{Análisis FODA}

El análisis FODA se realizó para la línea de artesanía textil, ya que en el diagnóstico se identificó que es la línea de artesanía que más se produce dentro de la Región, a continuación, se da a conocer las principales conclusiones:

- Poca capacidad de inversión e innovación tecnológica en los talleres de artesanía textil.

- Limitada innovación en técnicas, equipos, procesos, productos y diseños.

- Incremento de turistas en un $18 \%$ anual, que visitan la región Amazonas, generan mayor demanda de artesanías con un $61,4 \%$.

- Ubicación estratégica de los puntos de venta de artesanía, en los principales destinos turísticos de la región.

- Existencia de intermediarios y artesanos informales que compiten directamente con las asociaciones dedicadas a la confección de productos de artesanía.

\section{2. dentificación y Análisis de la Cadena Productiva de Artesanía Textil}

\section{- Bajo Nivel de Diseño e Innovación de Producto Artesanal}

Una de las grandes debilidades que tienen las asociaciones de artesanía textil, es la poca innovación y nuevos diseños de los productos, dicho punto crítico se logró identificar gracias a la pregunta del cuestionario donde se hace referencia si los artesanos utilizan o no maquinas o equipos para la elaboración de sus productos, en la cual la gran parte no cuenta con tecnología.

\section{- Falta de Identidad Cultural y Uso de Iconografía} Local

Para identificar dicho punto crítico se realizó visitas a campo, en cada asociación de artesanía donde se logró identificar sobre el poco uso de la iconografía local, esto también es debido a que la mayoría no cuenta con tecnología que facilite la elaboración de los productos con aplicación.

\section{-Bajo Nivel de Mejora de Proceso de Producción} Las asociaciones de artesanía textil de la región no manejan de una manera adecuada sus procesos de producción, no tienen en cuenta los tiempos de elaboración de un producto, así mismo desconocen como elaborar sus costos de producción, teniendo así un ingreso mínimo por cada producto.

\section{- Inadecuados Canales de Comercialización Artesanal.}

El bajo nivel de estrategias de promoción, marketing, publicidad y posicionamiento de la marca, hace que la venta de artesanía sea en poca cantidad; esto genera que las diferentes asociaciones de artesanía, su producción no sea constante logrando así que los artesanos no tengan una visión empresarial del negocio y con ello reduciendo sus fuentes de ingresos económicos, para la identificación de dicho punto crítico facilitó el análisis FODA donde se caracteriza la cadena de la artesanía de la Región.

\subsection{Desarrollo de Estrategias de Comercialización para Artesanía Textil}

Objetivo: Mejorar el nivel de competitividad de los artesanos y todos los agentes que participan en la cadena de valor de artesanía.

\section{a. Para Bajo Nivel de Diseño e Innovación de Producto Artesanal}

Estrategia 1: Fortalecimiento de capacidades en diseño e innovación del producto.

Estrategia 2: Fortalecimiento de capacidades sobre el aprovechamiento de recursos naturales como fibras y tintes para la producción e innovación de artesanía textil con identidad local. 


\section{b. Para Falta de Identidad Cultural y Uso de Iconografía Local}

Estrategia 1: Uso y aplicación de nuevos procesos y técnicas para la elaboración de sus productos, con el apoyo de las instituciones a través de un manual de procesos y un manual de uso y aplicación de iconografías para artesanía textil.

Estrategia 2: Desarrollo de un concurso de artesanía textil, mediante el uso y aplicación de iconografía local.

Estrategia 3: Fortalecimiento de capacidades sobre como rescatar y aprovechar técnicas ancestrales, colores y moda antigua con aplicación de iconografías de cada atractivo turístico de la zona.

\section{c. Para Bajo Nivel de Mejora de Proceso de Producción}

Estrategia 1: Gestión y Organización para poder canalizar financiamiento a través de proyectos para el mejoramiento e implementación con paquete tecnológico y articulación comercial.

Estrategia 2: Elaboración de un material de cocreación(manual) mediante la estandarización de procesos, así mismo desarrollar talleres sobre el uso y aplicación de estándares de calidad a productos de artesanía textil (Normas Técnicas).

Estrategia 3: Pasantías con otras regiones de país que exportan artesanía, para conocer como aplican los estándares de calidad.

Estrategia 4: Asistencia técnica en la mejora del uso del paquete técnico artesanal.

Estrategia 5: Implementación de las unidades de productoras de artesanía textil (talleres).

\section{d. Para Inadecuados Canales d Comercialización Artesanal.}

Estrategia 1: Fortalecimiento de capacidades en gestión empresarial y marketing corporativo.

Estrategia 2: Creación de una página Web.

Estrategia 3: Mejoramiento de la tienda artesanal Regional con la marca colectiva "La Rueca" en la Ciudad de Chachapoyas.

Estrategia 4: Estrategias de marketing corporativo usando las $4 \mathrm{P}$.

\section{DISCUSIÓN}

Los resultados obtenidos con respecto al primer objetivo específico, realizar un diagnóstico de la cadena productiva de artesanía en la región Amazonas, se obtuvo como resultado general que en la región Amazonas más del $80 \%$ se dedica a la artesanía textil (gorros, tejidos a cintura, morrales, chompas, etc), el $13 \%$ a la artesanía de madera (mesas de raíz, lámparas, sarcófagos, etc) y el $5 \%$ se dedican a la artesanía de bisutería (llaveros, aretes, collares, etc), dichas asociaciones elaboran sus productos de manera manual ya que el $78 \%$ no cuenta tecnología para la elaboración de sus productos, es decir no cuentan con un taller implementado con máquinas y equipos; y el $22 \%$ cuenta con tecnología para la elaboración de sus productos como máquina recta, remalladoras, taladros,. Así mismo los principales puntos de venta de artesanía están ubicados en los atractivos turísticos más visitados esto es debido a que cuando se habla de artesanía se relaciona de manera directa con el turismo, siendo la artesanía textil la que más se vende representada con un

$38.6 \%$, esto quiere decir que los productos textiles tienen gran acogida en el mercado, resaltando así que los principales clientes de artesanías son turistas nacionales $(60 \%)$ que al visitar los diferentes atractivos turísticos adquieren sus productos para llevar como un recuerdo de la región, los principales canales de comercialización son de manera directa ( Productor-consumidor) e indirecta( productorintermediario-consumidor) resultados que se asemejan a la tesis de Melchor, J. (2016) titulado plan de marketing para fomentar la exportación de artesanía textil a base de lana de oveja de la asociación APAGROP en el distrito de INCAHUASI hacia el mercado de Estados Unidos, quien en sus resultados obtuvo que ante la ausencia de estrategias que le permita dar a conocer su producto, posicionarlo e incrementar su cuota de mercado; dicha asociación no a logrado incrementar sus ventas, pero que a través del plan de marketing que es una herramientamuy importante que permitirá el crecimiento empresarial de la asociación de artesanos APAGROP, ya que le permitiría iniciarse en el comercio internacional y con ello ampliar su cuota de mercado.

Los resultados obtenidos con respecto al segundo objetivo, identificar y analizar los puntos críticos de cada eslabón de la cadena productiva de artesanía textil, de este contraste se puede decir que se identificó los principales puntos críticos de la cadena de valor, así mismo se realizó un análisis para mejorar dichos cuellos de botella encontrados como bajo nivel de diseño e innovación de producto artesanal en la línea textil, también se encontró falta de identidad cultural que representa la poca representación de cultura y aplicación de icnografía que tienen sus productos textiles, además se identificó el bajo nivel de mejora de proceso de producción. Finalmente, inadecuados canales de comercialización artesanal, esto es debido a que los artesanos(as) desconocen temas de marketing, publicidad, etc para ofertar sus productos, estos resultados se asemejan a la tesis de Tesén, D. (2016) titulado Estrategias de marketing 
para fomentar la exportación de artículos de regalo y decoración de lana de ovino: Caso asociación de artesanos productores agropecuarios - APAGROP, de Incahuasi al mercado de Finlandia, quien obtuvo como resultado que la asociación no cuenta con estrategias de marketing y ventas lo cual ha impedido su posicionamiento en el mercado, ello se ve reflejado en sus volúmenes de ventas, los cuales son bajos y en ocasiones nulos.

Los resultados obtenidos con respecto al tercer objetivo, desarrollar estrategias para mejorar los canales de comercialización de la artesanía textil, se desarrollaron estrategias para mejorar los

diferentes puntos críticos identificados, uno de ellos con respecto a los inadecuados canales de comercialización para ello se desarrolló estrategias haciendo uso del marketing corporativo con las $4 \mathrm{p}$, así mismo capacitaciones para los artesanos(as) en temas de marketing, gestión empresarial, mejoramiento de la tienda artesanal con la marca colectiva la Rueca, etc. Estos resultados guardan semejanza con la investigación realizada por Quiñones, Z. (2017) en su tesis titulada plan de Marketing para la participación de una Asociación de Artesanos Nacionales en Ferias Internacionales; quien concluyó que en la propuesta se ha diseñado un plan de marketing para la asociación de artesanos Asamil, con la finalidad de posicionarse en el mercado internacional e incrementar sus ventas de artesanía.

\section{CONCLUSIONES}

Según el diagnóstico de la cadena productiva de artesanía en la Región; se identificó 4 líneas principales de artesanía en la región como son artesanía textil, madera, bisutería y cerámica, siendo la artesanía textil la que más se trabaja, asimismo se caracterizaron las asociaciones legalmente registradas que están integradas en su mayoría por mujeres. Los principales puntos de venta están ubicados en los atractivos turísticos más visitados esto es debido a que cuando se habla de artesanía se relaciona de manera directa con el turismo, los principales compradores de artesanía son de procedencia nacional, esto se debe a que la gran mayoría son turistas quienes durante su visita a los atractivos turísticos de la región adquieren artesanías.

Se identificaron dentro de la cadena productiva de artesanía textil; bajo nivel de diseño e innovación de producto artesanal en la línea textil, falta de identidad cultural y uso de iconografía local, bajo nivel de mejora deproceso de producción e inadecuados canales de comercialización artesanal.

Se desarrollaron estrategias como; capacitaciones en diseño e innovación, manual de uso y aplicación de iconografías para artesanía textil, manual de proceso para artesanía textil, estrategias haciendo uso del marketing corporativo con las $4 \mathrm{p}$, así mismo capacitaciones para los artesanos(as) en temas de marketing, gestión empresarial, mejoramiento de la tienda artesanal con la marca colectiva la Rueca, etc. nes.

\section{REFERENCIAS BIBLIOGRÁFICAS}

Abreu, J. L. (2014). El Método de la Investigación. Obtenido d http://www.spentamexico.org/v9n3/A17.9(3)195-204.pdf

Centro de Innovación Tecnológica de Artesanía y Turismo (2018). Perfil delConsumidor de Artesanía en la Región Amazonas. Chachapoyas.

Hernández, F. (2003). Metodología de la Investigación. Obtenido d e http://catarina.udlap.mx

Melchor Panta, J. A. (2016). Plan de marketing para fomentar la exportación de artesanía textil a base de lana de oveja de la asociación APAGROP en el distrito de Incahuasi hacia el mercado de Estados Unidos. Obtenido dehttp://repositorio.uss.edu.pe/bitstream/ handle/uss/3124/MELCHOR\%20PAN TA.pdf? sequence $=7 \&$ isAllowed $=\mathrm{y}$

Ministerio de Comercio Exterior y Turismo (2018). Agencia Peruana de Noticias. Obtenido de https://andina.pe/agencia/noticiamincetur-potenciara-turismo- amazonas-caraal-bicentenario- 733199.aspx

Quiñones Seguil, Z. M. (2017). Plan de Marketing para la participación de una Asociación de Artesanos Nacionales en Ferias I n t e r n a c i o n a l e s. Ob te n i d o dehttp://repositorio.uwiener.edu.pe/bitstre am/handle/123456789/511/T061 7376 0929_T.pdf?sequence $=2 \&$ isAllowed $=\bar{y}$

Tesén Martino, D. A. (2016). Estrategias de marketing para fomentar la exportación de artículos de regalo y decoración de lana de ovino: Caso asociación de artesanos productores agropecuarios - APAGROP. Obtenido d http://repositorio.uwiener.edu.pe/bitstre $\mathrm{am} / \mathrm{handle} / 123456789 / 511 / \mathrm{T} 0617376$ 0929 T.pdf? sequence $=2 \&$ is Allowed $=y$ 277.pdf? sequence $=1 \&$ is Allowed $=y$ 